UDC: $811.134 .2^{\prime} 27$

DOI: https://doi.org/10.18485/hispserb.2019.2.ch8

\author{
Jelena Kovač ${ }^{1}$ \\ Universidad de Priština con sede en Kosovska Mitrovica \\ Serbia
}

\title{
MODELOS CULTURALES COGNITIVOS Y LA CORTESÍA EN EL DISCURSO ACADÉMICO: ESTUDIO DE CASO ENFOCADO EN LA POBLACIÓN ESTUDIANTIL UNIVERSITARIA
}

\begin{abstract}
Resumen
La cortesía representa una faceta importante de las interacciones sociales, especialmente en situaciones donde hay una distribución desigual del poder en el discurso. El objetivo de este trabajo es investigar el discurso académico entre los profesores universitarios y los estudiantes de estudios de doctorado en Serbia y España, con en el enfoque puesto en los principios de la cortesía que se utilizan en la comunicación mutua como reflejo de modelos culturales cognitivos. Teniendo en cuenta que el discurso académico contiene una relación vertical entre los participantes, se parte de la hipótesis de que los estudiantes no tienen mucho poder en este proceso. A través de un análisis interdisciplinario comparativo del discurso académico basado en los postulados de la sociolingüística, la antropología cognitiva y el análisis crítico del discurso, se intentan descubrir modelos culturales cognitivos que conducen a una situación relativa de los participantes en la comunicación académica, así como a la desigualdad social en el fondo del uso de la cortesía. Como procedimiento metodológico para la investigación, ha sido seleccionado el estudio de caso. El método de recolección de datos utilizado ha sido la asistencia a clases de

${ }^{1}$ kovac.jelena@gmail.com
\end{abstract}


los estudios de doctorado donde se han llevado a cabo varias observaciones $\mathrm{y}$ entrevistas semiestructuradas con los estudiantes. El objetivo era que los resultados de la investigación muestren si las comunidades académicas serbia y española comparten los mismos modelos culturales cognitivos y el mismo grado de cortesía, y si una comunidad es más tradicional que la otra.

Palabras clave: cortesía, modelos culturales cognitivos, discurso académico, análisis interdisciplinario.

\section{Introducción}

Cuando se trata de la relación entre el idioma y sus usuarios, se requiere el uso de diferentes elementos y estructuras para que se logre una comunicación eficaz entre los interlocutores y para que la transmisión del mensaje sea eficaz. No importa qué tipo de discurso sea, durante la conversación uno debe tener en cuenta la manera de comunicar el mensaje, de pedir información, de quién es su interlocutor, etc.

En el foco de este trabajo se encuentra la cortesía en el discurso académico como reflejo de modelos culturales cognitivos. La cortesía representa una faceta importante de las interacciones sociales, especialmente en situaciones donde hay una distribución desigual del poder en el discurso. Cuando se trata del discurso académico, este tipo de discurso refleja relaciones de poder desiguales durante la comunicación, donde la lengua se convierte en un medio para preservar la relación de desigualdad (ver Fairclough 2001) y donde los profesores están en posiciones más altas de poder mientras que los estudiantes tienen menos poder durante el proceso en sí (Filipović 2009: 46-47).

El objetivo de este trabajo es investigar el discurso académico entre los profesores universitarios y los estudiantes de estudios de doctorado en Serbia y España (Universidad de Belgrado y Universidad de Granada), con el enfoque puesto en los principios de la cortesía que se utilizan en la comunicación mutua como reflejo de modelos culturales cognitivos. A los efectos de este trabajo, se hace hincapié en el discurso de la población estudiantil universitaria. Tomando en cuenta que el discurso académico contiene una relación vertical entre los participantes, o sea que en el discurso académico existe cierta jerarquía, se parte de la hipótesis de que los estudiantes no poseen un alto grado de poder en este proceso. También, el objetivo es comparar dos contextos socioculturales, sus similitudes y diferencias, y extender el debate sobre modelos cognitivos culturales al 
campo académico. Se supone que dentro de la comunidad académica están presentes modelos culturales cognitivos tradicionales, es decir, los modelos que definen claramente cómo debe posicionarse cada uno de los participantes en el discurso, utilizando las estructuras de la cortesía como un principio que regula y equilibra la distancia social (Leech 1983: 83).

A través de un análisis interdisciplinario comparativo del discurso académico, basado en los postulados de la sociolingüística, la antropología cognitiva y el análisis crítico del discurso, se intentan descubrir modelos culturales cognitivos que conducen al empleo de los principios de la cortesía.

Como procedimiento metodológico para la investigación ha sido seleccionado el análisis sociolingüístico cualitativo: estudio de caso. El método de recolección de datos utilizado ha sido la asistencia a clases en los estudios de doctorado donde se han llevado a cabo varias observaciones y entrevistas semiestructuradas con los estudiantes.

Se esperaba que los resultados de la investigación muestren si la población estudiantil universitaria serbia y española comparten los mismos modelos culturales cognitivos y el mismo grado de la cortesía, así como si una comunidad es más tradicional que la otra.

\section{Marco teórico}

Cuando hablamos sobre los modelos culturales cognitivos y la cortesía, cabe destacar que ambos conceptos, tanto los modelos culturales como la cortesía, son conjuntos de conocimiento y normas sociales que regulan el comportamiento de las personas en la sociedad para funcionar con éxito (ver Holland \& Quinn 1995; Escandell Vidal 2006; Filipović 2009). Precisamente por esta razón se considera importante asociar estos términos con la expectativa de que el discurso académico muestre que los modelos culturales y los principios de la cortesía se correlacionan significativamente entre sí.

\subsection{La cortesía, la cultura y la lengua en la ciencia}

En los enfoques críticos que se centran en cómo las relaciones sociales se experimentan en un idioma, la lengua no es solo un medio de expresión o comunicación, sino que es una práctica que construye relaciones entre las personas, y que es construida por los propios hablantes. A través del idioma, los hablantes se comprenden a sí mismos, a su entorno social, sus posibilidades y su perspectiva social (Norton \& 
Toohey 2004: 1). En vista del hecho de que la conversación no siempre involucra a interlocutores que tienen una relación de igualdad, que no comparten los mismos objetivos de comunicación, ni son objeto de un tema de discusión adecuado para hablar de ello de manera abierta, de una manera completamente directa, se recurre a ella por medios indirectos de expresión del mensaje, que se logra precisamente a través de los principios de la teoría de la cortesía (Brown \& Levinson 1987).

El concepto clave en la teoría de la cortesía de Penelope Brown y Stephen C. Levinson (1987) es el concepto de imagen pública (en ingles face). El concepto mencionado sugiere que, al comunicarse con la gente, la imagen pública de los participantes en la conversación, por un lado, puede estar amenazada, y por otro lado puede estar salvada durante la conversación. Además, la imagen pública se manifiesta a través de la imagen que el individuo quiere que otros miembros de la sociedad tengan sobre él (ver Goffman 1967; Brown \& Levinson 1987).

Dado que la lengua forma parte integral de la cultura y la sociedad, la relación entre ellas, es decir, la interacción entre la lengua misma, los pensamientos y la percepción del mundo, representa un lugar importante en la ciencia lingüística (Filipović 2009: 110). Alessandro Duranti (2003: 323) señala que en la antropología estadounidense la lengua se entiende como una cultura, que estos dos conceptos no están separados y que la lengua se usa en ciertos contextos como un evento del habla. Según Dell Hymes (1980: 16-17), los valores y creencias culturales, roles e instituciones sociales, historia social y ecología de una comunidad deben ser examinados con respecto a su posición en eventos y patrones comunicativos, tal como son, igual que cada aspecto de la vida social puede ser estudiado. El código de idioma, o solo el habla, no debe considerarse un conjunto completo de datos, sino que debe tener en cuenta la comunidad social que desempeña el papel del contexto.

Cuando hablamos sobre la sociolingüística crítica, esta disciplina científica trata de determinar cómo están conectadas las relaciones sociales y la práctica lingüística, o cómo la práctica lingüística "contribuye a la desigualdad social y al desequilibrio en la distribución del poder social", explorando el uso de la lengua, las variaciones lingüísticas y las actitudes de los hablantes de acuerdo con el discurso (Filipović 2009: 135).

En cuanto al análisis crítico del discurso, su postulado es, como señala Norman Fairclough (2001: 27), que la lengua se está convirtiendo en uno de los medios legítimos de preservar la relación de desigualdad en la comunidad social. La universidad como una organización social con poder está estructurada de manera firme y homogénea con roles y 
funciones definidas y elaboradas con precisión. El poder social en grupos y organizaciones se basa en los principios de jerarquía (Pešić \& Bazić 2008: 245-246).

\section{La investigación}

A los efectos del presente trabajo, se presenta un estudio de caso cualitativo que pretende proporcionar información sobre los modelos culturales cognitivos y los principios de la cortesía en el discurso académico. La metodología cualitativa es la más adecuada para investigar y presentar los fenómenos en el foco de este trabajo, ya que es importante que estos fenómenos sean percibidos y descritos desde la perspectiva de los participantes en la investigación.

En el foco de la investigación está la población estudiantil de Serbia y España (Universidad de Belgrado (Facultad de Filología) y Universidad de Granada). Todos los estudiantes que han participado en la investigación son doctorandos.

\subsection{Metodología y corpus}

La investigación cualitativa aquí expuesta se realizó durante mayo / junio de 2017 (en la Universidad de Granada, España) y desde marzo de 2018 hasta abril de 2019 (en la Universidad de Belgrado, Facultad de Filología, Serbia) e involucró a ocho encuestados (tres de España y cinco de Serbia). Se llevaron a cabo siete observaciones de clases / seminarios para doctorandos (cuatro en España durante el seminario para doctorandos y tres en Serbia durante las clases). En ambas universidades primero se llevaron a cabo las observaciones de clases / seminarios y luego se hicieron entrevistas semiestructuradas con los doctorandos que asistieron a las clases observadas.

La guía para la entrevista semiestructurada consistía en veinte preguntas y cada una de las entrevistas con los doctorandos duraba entre veinte minutos y una hora. Las preguntas abarcaban tanto la comunicación oral como la escrita (los correos electrónicos que son una forma generalizada de comunicación con profesores hoy en día). Los doctorandos no sabían de antemano lo que se les iba a preguntar.

Para resumir, el corpus de la investigación lo componen todos los registros recopilados del discurso espontáneo durante las clases / sesiones en el seminario (observaciones), ejemplos de las entrevistas semiestructuradas, así como la interpretación del discurso académico. 


\subsection{Los resultados de la investigación}

Sobre la base de las observaciones realizadas en ambas universidades (las sesiones del seminario para doctorandos / las clases de doctorado), se concluyó que en ambos países existe un discurso académico igual o similar en el aula por parte de los doctorandos. El contexto del aula es sin duda formal, donde cada participante se adhiere a las reglas de conducta válidas en la comunidad académica. En ambas comunidades, la mayoría de los doctorandos no participa activamente en los debates ni responde a las preguntas de los profesores cuando los profesores las hacen en general. Las razones de este comportamiento están fuera del alcance de esta investigación, por lo tanto, no las vamos a analizar en este trabajo. Cuando se dirigen a los profesores en el aula, los doctorandos serbios y españoles empelan el pronombre personal usted. Sin embargo, hay una excepción, pero solamente en los casos de doctorandos españoles. Esta excepción está relacionada con una faceta extralingüística - la edad. Durante las dos sesiones en el seminario de los doctorandos en la Universidad de Granada, las clases las impartía un profesor de treinta y nueve años, así que todos los doctorandos empleaban el pronombre tú. En Serbia no se ha encontrado ese uso. Lo mencionado nos lleva a la conclusión de que, en España, en ciertos casos, se puede notar el modelo cultural cognitivo de modernización, o sea, el cambio de la trayectoria tradicional del discurso académico.

En cuanto a las entrevistas semiestructuradas, se han notado ciertas diferencias, especialmente en los casos que se refieren al uso de los pronombres personales tú / usted, lo que ya hemos notado durante las observaciones. Como se ha señalado al comienzo de la sección, la guía para la entrevista contiene veinte preguntas en total que cubren la comunicación oral y escrita (hay una versión en serbio y en español). Cada entrevista semiestructurada con los doctorandos duró entre veinte minutos y una hora. Los doctorandos recibieron información sobre el tema y el propósito de la investigación, sin embargo, no sabían de antemano qué preguntas se les iba a preguntar.

Las primeras cinco preguntas se referían a la noción de cortesía en general, y luego a la cortesía en la comunicación académica / el contexto académico, a la organización de la comunidad académica, así como a los límites de la comunicación entre profesores y estudiantes. Las preguntas sexta y séptima se relacionan con la forma en que debería aparecer una comunicación oral y escrita entre profesores y estudiantes en sentido formal e informal. Una de las preguntas fue sobre si la comunicación y 
la manera de dirigirse a los profesores deberían cambiar con la edad del profesor y con el logro de títulos académicos superiores. Las siguientes preguntas se relacionaron con la forma en que el contexto (durante la enseñanza / actividades extracurriculares) determina y dirige las reglas de comunicación, cómo debe ser la comunicación con los estudiantes de los estudios de doctorado, cómo se dirigen los profesores a los estudiantes de grado / máster / doctorado. Las similitudes en las respuestas de los doctorandos serbios y españoles son muy grandes con respecto a todos los temas, por lo que hay un nivel muy alto de coherencia entre ellos. Todos los entrevistados están de acuerdo en que la cortesía es necesaria tanto en el contexto académico como en el cotidiano. Hubo preguntas donde surgieron diferencias en el entorno académico entre los doctorandos serbios y los españoles. Esas preguntas son las siguientes: ¿Te dijo alguna vez un / una profesor / a que lo / la llamaras por su nombre y que lo / la tutearas?, ¿Te sorprende cuando el / la profesor / a te trata de tú?, ¿Es esa una práctica habitual en tu universidad? Los doctorandos serbios respondieron que no les sorprendía cuando los profesores utilizaban el pronombre tú, pero que nunca les había pasado que los profesores propusieran el empleo del pronombre tú. Los doctorandos entrevistados serbios siempre utilizan usted cuando se dirigen a sus profesores, a diferencia de los españoles que usan tú incluso cuando el profesor es mayor (esa propuesta viene previamente de parte del profesor).

\section{Conclusiones}

Durante la comunicación, todos los hablantes observan consciente o inconscientemente ciertas normas convencionales de conducta verbal, que surgen de sus modelos culturales cognitivos, de modo que se pueden distinguir dos principios básicos en la conversación. Se dice que es necesario cooperar en la comunicación con el interlocutor, y que, en consecuencia, se expresa la medida de persistencia menos necesaria (Bugarski 1995: 149), que también se refiere a la comunicación dentro de la comunidad académica que ha sido el foco de este trabajo.

Los resultados de la investigación han mostrado que el modelo cultural cognitivo tradicional y de jerarquía es el más frecuente en el discurso académico. En el caso de ambas comunidades el modelo cultural cognitivo tradicional está presente en la mayoría de situaciones comunicativas. Sin embargo, el discurso académico de los doctorandos españoles hacia los profesores que conocen desde antes es menos formal que el discurso de los doctorandos serbios en cuanto al empleo de los 
pronombres personales tú / usted y la manera de saludar hola en vez de buenos días / buenas tardes.

No obstante, la conclusión general que surge de esta investigación es que no se han encontrado diferencias cualitativas en las formas de comunicación en las comunidades académicas serbia y española (por lo menos en el caso de los doctorandos que participaron en la investigación). Las conclusiones resultantes ponen de manifiesto que en Serbia (Facultad de Filología, Universidad en Belgrado) y España (Universidad de Granada) están vigentes los mismos o similares modelos culturales cognitivos.

\section{BIBLIOGRAFÍA}

Brown, Penelope y Stephen C. Levinson. Politeness: Some Universals in Language Usage. UK: Cambridge University Press, 1987. Print.

Bugarski, Ranko. Uvod u opštu lingvistiku. Beograd: Zavod za udžbenike i nastavna sredstva, 1995. Štampano.

Duranti, Alessandro. "Language as Culture in U.S. Anthropology; Three Paradigms". Current Anthropology, 44 (2003): 323-347. Print.

Escandell Vidal, María Victoria. Introducción a la pragmática. Barcelona: Ariel Lingüística, 2006. Impreso.

Fairclough, Norman. Language and Power. $2^{\text {nd }}$ edition. Essex: Pearson Education Limited, 2001. Print.

Filipović, Jelena. Moć reči. Ogledi iz kritičke sociolingvistike. Beograd: Zadužbina Andrejević, 2009. Štampano.

Goffman, Erving. Interaction ritual; essays on face-to-face behavior. Garden City, N.Y: Doubleday, 1967. Print.

Hajmz, Del. Etnografija komunikacije. Beograd: Beogradski izdavačkografički zavod. Biblioteka XX vek, 1980. Štampano.

Holland, Dorothy \& Naomi Quinn. Cultural Models in Language and Thought. Cambridge: Cambridge University Press, 1995. Print.

Leech, Geoffrey N. Principles of Pragmatics. London / New York: Longman, 1983. Print.

Norton, Bonny \& Kelleen Toohey. Critical Pedagogies and Language Learning. UK: Cambridge University Press, 2004. Print.

Pešić, Mihailo \& Jovan Bazić. Sociologija. Beograd: Fakultet za turistički i hotelijerski menadžment, Univerzitet Singidunum, 2008. Štampano. 


\section{COGNITIVE CULTURAL MODELS AND THE POLITENESS IN THE ACADEMIC DISCOURSE: \\ CASE STUDY FOCUSED ON THE UNIVERSITY STUDENT POPULATION}

\section{Summary}

The politeness represents an important facet of social interactions, especially in situations where there is an unequal distribution of power in discourse. The aim of this paper is to investigate the academic discourse between university professors and students of doctoral studies in Serbia and Spain, with a focus on the politeness principles which are used in mutual communication as a reflection of cognitive cultural models. Taking into account that the academic discourse contains a vertical relation among the participants, it is assumed that students do not have much power in this process. Through a comparative interdisciplinary analysis of academic discourse based on the postulates of sociolinguistics, cognitive anthropology and the critical analysis of discourse, we try to discover cognitive cultural models that lead to the relative situation of participants in academic communication, as well as the social inequality in the background of the politeness usage. As a methodological procedure for the investigation, the case study has been selected. The methods of data collection are assistance to classes in doctoral studies where several observations and semi-structured interviews with the students are carried out. The results of the research are expected to show whether the Serbian and Spanish academic communities share the same cognitive cultural models and the same degree of politeness, and whether one community is more traditional than the other.

Keywords: politeness, cognitive cultural models, academic discourse, interdisciplinary analysis. 